\title{
Contrast and glare sensitivity in epilepsy patients treated with vigabatrin or carbamazepine monotherapy compared with healthy volunteers
}

\author{
Iiris Nousiainen, Reetta Kälviäinen, Maija Mäntyjärvi
}

\begin{abstract}
Backgroundlaim-Many antiepileptic drugs have influence on visual functions. The aim of this study was to investigate possible changes in contrast sensitivity, macular photostress, and brightness acuity (glare) tests in patients with epilepsy undergoing vigabatrin (VGB) or carbamazepine (CBZ) monotherapy compared with healthy volunteers.

Methods-32 patients undergoing VGB therapy, 18 patients undergoing CBZ therapy, and 35 healthy volunteers were asked to participate in an ophthalmological examination. In the previous study, visual field constrictions were reported in $40 \%$ of the patients treated with VGB monotherapy. In the present study, these VGB and CBZ monotherapy patients were examined for photopic contrast sensitivity with the Pelli-Robson letter chart and brightness acuity and macular photostress with the Mentor BAT brightness acuity tester.
\end{abstract}

Results-Contrast sensitivity with the Pelli-Robson letter chart showed no difference between these groups and normal subjects (ANOVA: $\mathrm{p}=0.534$ in the right eye, $p=0.692$ in the left eye) but the VGB therapy patients showed a positive correlation between the contrast sensitivity values and the extents of the visual fields in linear regression $(R=0.498, p=0.05$ in the right eye, $R=0.476, p=0.06$ in the left eye). Macular photostress and glare tests were equal in both groups and did not differ from normal values.

Conclusion-The results of this study indicate that carbamazepine therapy has no effect on contrast sensitivity. Vigabatrin seems to impair contrast sensitivity in those patients who have concentrically constricted in their visual fields. Neither GBZ nor VGB affect glare sensitivity. (Br F Ophthalmol 2000;84:622-625)

Carbamazepine (CBZ) is a standard antiepileptic drug for partial and generalised tonicclonic seizures. Its mechanism of action is to inhibit high frequency neuronal firing by blocking the voltage gated sodium channel. Visual disturbances such as nystagmus, diplopia, and blurred vision are well known adverse effects of CBZ. ${ }^{1}$ Abnormal contrast sensitivity and enhanced glare sensitivity have been reported earlier in patients undergoing CBZ therapy. $^{2-4}$
$\gamma$-Aminobutyric acid (GABA) is an inhibitory neurotransmitter in the central nervous system (CNS) as well as in horizontal, amacrine, bipolar, and ganglion cells of the retina. ${ }^{5-7}$ Vigabatrin ( $\gamma$-vinyl-GABA, VGB) is a selective irreversible inhibitor of GABA transaminase. Inhibition of GABA transaminase produces greater available pools of presynaptic GABA for release in CNS synapses. Vigabatrin has both antiseizure and antiepileptogenic properties with few adverse effects. ${ }^{89}$ Currently, VGB is approved in over 60 countries for the adjunctive management of partial epilepsy not satisfactorily controlled by conventional therapy and for initial monotherapy in the management of infantile spasms.

Spontaneous cases of bilateral concentric constriction of visual fields have recently been described in connection with VGB therapy. ${ }^{10-14}$ In a case report on three VGB therapy patients, Krauss et $a l^{15}$ described changes in the electroretinogram, suggesting bilateral retinal dysfunction. Photopsia and scintillation have been described as subjective ocular side effects of vigabatrin. ${ }^{16}$ However, it has also been observed that add-on therapy with VGB reduces impairment of contrast sensitivity caused by CBZ therapy. ${ }^{17}$ The study of Steinhoff et $a l^{18}$ showed no alterations in perception thresholds for gratings in epilepsy patients undergoing $\mathrm{CBZ}$ or combined CBZ and VGB therapy. A single dose of VGB did not change spatial contrast sensitivity measured with a monitor system. ${ }^{19} 20$

We have described earlier that VGB monotherapy patients have concentric constricted visual fields compared with CBZ monotherapy patients. ${ }^{21}$ The purpose of this study was to investigate contrast sensitivity, macular photostress, and glare sensitivity in epilepsy patients undergoing VGB or CBZ monotherapy. Furthermore, our aim was to determine the occurrence of eventual abnormal visual functions and their correlation with the visual field constriction observed in VGB patients.

\section{Patients}

Thirty two VGB monotherapy patients (aged 19-73 years, mean 40.4 (SD 6.3)) and 18 CBZ monotherapy patients (aged 20-70 years, mean 43.8 (18.1)) were examined at the department of ophthalmology, Kuopio University Hospital, Kuopio, Finland. The patients had been randomised initially for a VGB $v$ CBZ monotherapy study during the years $1988-95,,^{162}$ and they still continued their monotherapy successfully. The time of VGB 
therapy varied from 29 to 119 months (mean 68.7 (29.5)) and the daily dosage of VGB from 2 to $4 \mathrm{~g}$. The cumulative dose of VGB was 1800 to $14160 \mathrm{~g}$ (mean 5570 (3010) g). The duration of CBZ therapy varied from 32 to 108 months (mean 60.5 (26.2)). The plasma concentration of CBZ was in the therapeutic range $(20-50 \mu \mathrm{mol} / \mathrm{l})$. All patients had well controlled partial epilepsy and had been seizure free for at least 1 year. Thirty five healthy volunteers (aged 20-72 years, mean 45.9 (16.0)) were examined to produce the normal values of the Pelli-Robson test. The ages of the patients and controls were similar (ANOVA, $\mathrm{p}=0.441$ ).

This study was reviewed and accepted by the ethics committee of the university and the University Hospital of Kuopio (Finland) and was performed in accordance with the ethical standards of the 1964 Declaration of Helsinki. Written informed consent was obtained from all the patients.

\section{Methods}

To exclude other ophthalmic disorders a full ophthalmological evaluation was performed. The ophthalmological examination consisted of a history of visual symptoms, testing the best corrected visual acuity, phorias and tropias, biomicroscopy, and direct and indirect ophthalmoscopy with the aid of tropicamide $0.8 \%$-phenylephrine $5 \%$ drops. Intraocular tension was measured with the Goldmann applanation tonometer. Visual fields were examined with the kinetic Goldmann perimeter (Haag-Streit, Bern, Switzerland) using objects $I V / 4$ in the peripheral and I/3 in the central isoptres.

Two right eyes, one aphakic and one microphthalmic, were excluded from the study in the VGB group. In our previous study, ${ }^{21}$ we found, with kinetic Goldmann perimetry, severely constricted visual fields (temporal extent narrower than $50^{\circ}$ ) in three and mildly constricted visual fields (temporal extent narrower than $70^{\circ}$ ) in 10 VGB patients. Nineteen patients had normal visual fields. All CBZ patients had normal visual fields. There were no other ocular abnormalities in either group.

Photopic contrast sensitivity was measured monocularly with the Pelli-Robson letter chart (Clement Clarke International Ltd, London, Fig 1) with the patient's own distance correction. The standard luminance of the test chart was $100 \mathrm{~cd} / \mathrm{m}^{2}$ and the test distance 3 metres, corresponding to the spatial frequency of $3 \mathrm{cpd}$ (cycles per degree). The Pelli-Robson contrast sensitivity test is found to be a short and reliable test for clinical use. ${ }^{22} 23$ It consists of two groups of three letters (triplets) per row, with the two groups differing in contrast. The contrast of the groups decreases by approximately $0.15 \mathrm{log}$ units, ranging from a contrast of 1 or $100 \%$ at the upper left corner to a contrast of 0.006 or $0.6 \%$ at the lower right corner of the chart. Patients were required to read the letters from high to low contrast. The last triplet where at least two of the three letters were named correctly was accepted for the contrast value. In the results, the values of logarithmic contrast sensitivity (1/contrast) are given.

Macular photostress and glare sensitivity were measured monocularly with the Mentor BAT brightness acuity test (Mentor O\&O, Inc, Norwell, MA, USA, Fig 2). In the brightness acuity test, the distant visual acuity with the best correction was measured without glare and with low, intermediate, and high glare (41, 342 , and $1370 \mathrm{~cd} / \mathrm{m}^{2}$ ). Normal observers have the same visual acuity in low and intermediate

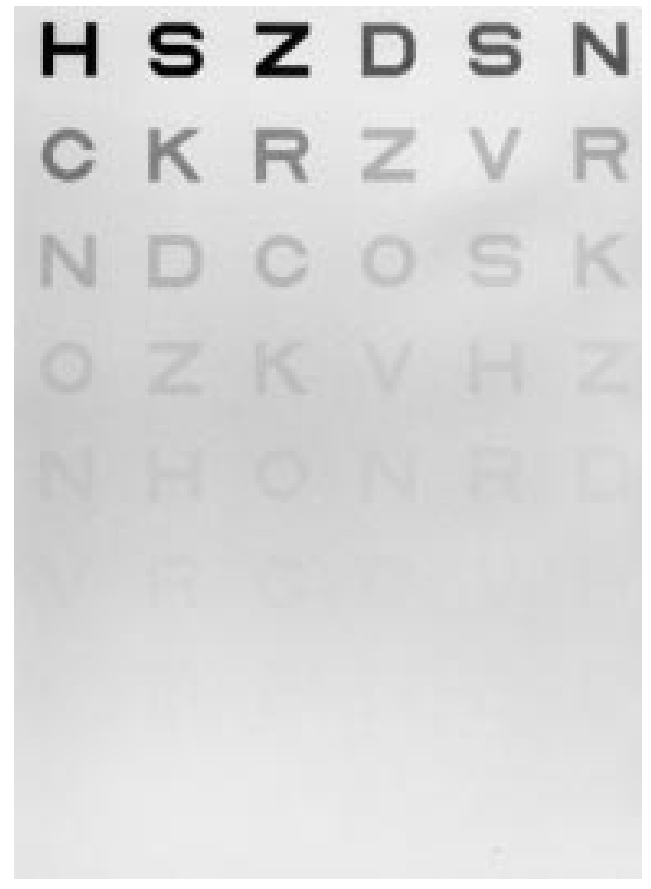

Figure 1 Pelli-Robson letter chart.

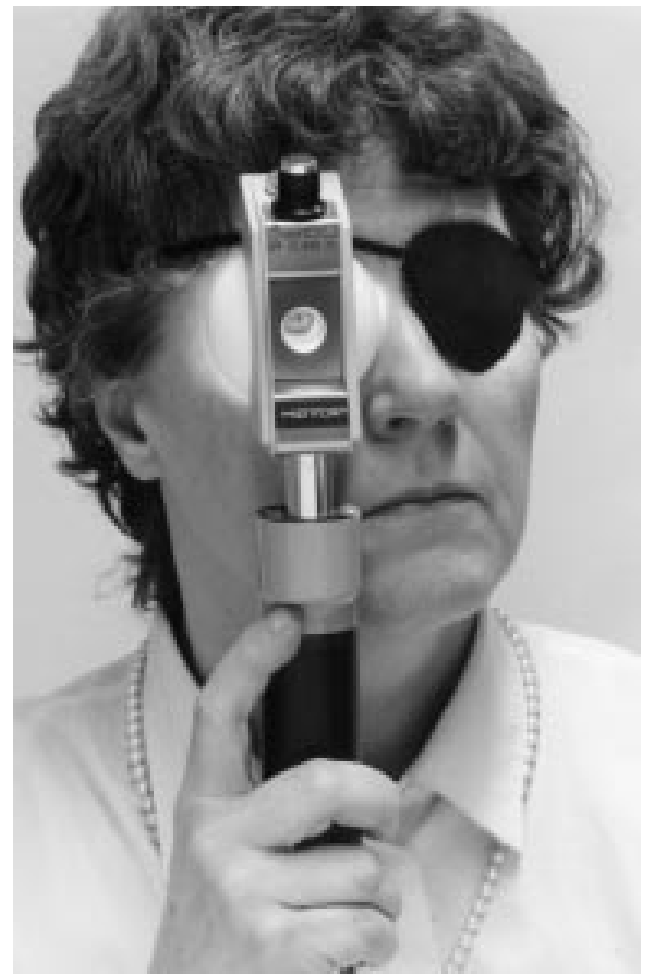

Figure 2 Brightness acuity testing with BAT. 
Table 1 Contrast sensitivity with Pelli-Robson letter chart in patients treated with vigabatrin or carbamazepine monotherapy and normal controls (mean (SD)). (ANOVA, $p<0.05$ significant)

\begin{tabular}{lllll}
\hline & $\begin{array}{l}\text { Vigabatrin } \\
(n=30 R E, 32 ~ L E)\end{array}$ & $\begin{array}{l}\text { Carbamazepine } \\
(n=18)\end{array}$ & $\begin{array}{l}\text { Normal controls } \\
(n=35)\end{array}$ & $p$ Value \\
\hline Right eye (RE) & $1.76(0.14)$ & $1.78(0.11)$ & $1.77(0.14)$ & 0.534 \\
Left eye (LE) & $1.74(0.19)$ & $1.78(0.13)$ & $1.80(0.12)$ & 0.692 \\
\hline
\end{tabular}

brightness as they do without glare. At high brightness, the visual acuity can be two Snellen lines lower than it is without glare but is usually the same or even one Snellen line better owing to the constricted pupil. In the macular photostress test, high glare was maintained for 15 seconds, and the length of time required for the visual acuity to recover to two lines above the line of the best corrected visual acuity was recorded. According to Glaser et $a l^{4}$ the normal recovery time ranges from 0 to $30 \mathrm{sec}-$ onds.

The dark adaptation examination was done monocularly with the Goldmann-Weekers Adaptometer (Haag-Streit, Bern, Switzerland) according to the instructions for the absolute threshold. We accepted as normal values studied in our clinic 3.8 to $4.6 \log$ units (mean 4.2

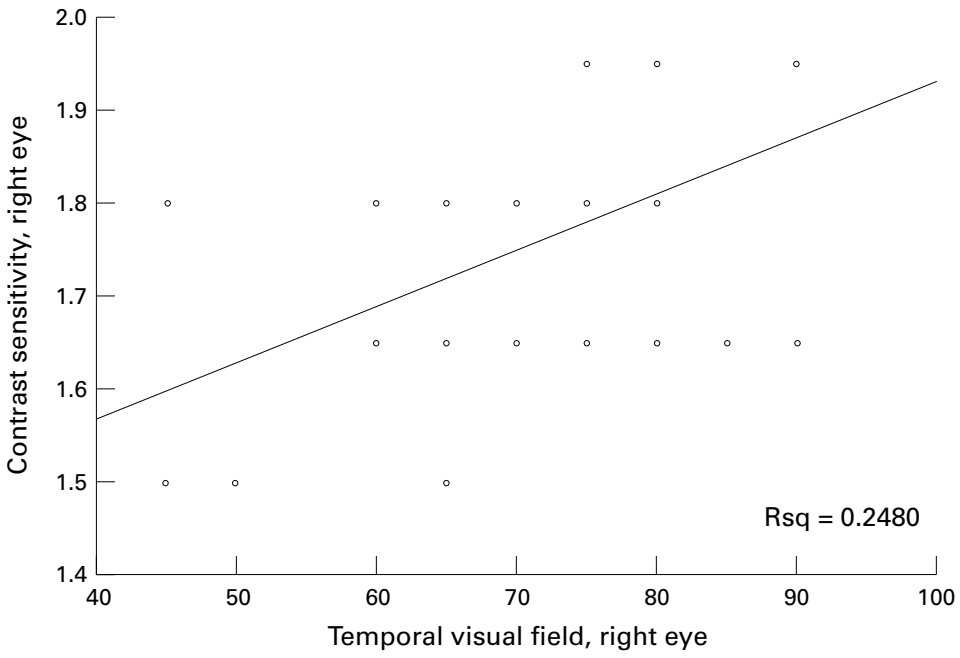

Figure 3 Comparison between contrast sensitivity and the extents of the temporal visual fields (in degrees) in the VGB therapy patients by linear regression. Right eye.

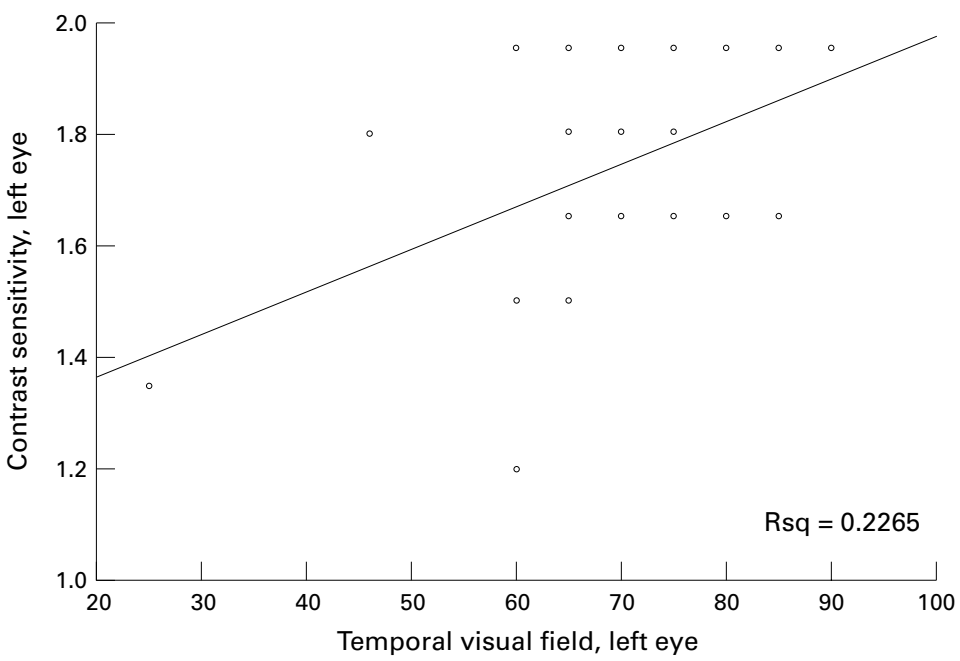

Figure 4 Comparison between contrast sensitivity and the extents of the temporal visual fields (in degrees) in the VGB therapy patients by linear regression. Left eye.
(SD 0.2)) and 5-9 minutes (mean 7 (1.0)) for the cone thresholds and times and 1.8 to 2.4 $\log$ units (mean $2.13(0.15)$ ) and 22-32 minutes (mean 27.02 (2.53)) for the final rod thresholds and times. The luminance of $2 \mathrm{log}$ units corresponds to $0.00001 \mathrm{~cd} / \mathrm{m}^{2}$.

Statistical analyses were performed with the SPSS programs using one way analysis of variance (ANOVA) with Duncan's post hoc test and linear regression. A p value $<0.05$ was considered statistically significant.

\section{Results}

The visual acuities of both eyes were normal, $6 / 7.5(\log$ MAR +0.1$)$ or better in all three groups. In the VGB group, the refraction of the right eye (in spherical equivalents) varied from -3.5 dioptres (D) to $+2.5 \mathrm{D}$ (mean -0.31 (1.42)) and that of the left eye from $-3.5 \mathrm{D}$ to $+2.5 \mathrm{D}$ (mean -0.39 (1.27)). In the CBZ group, the refraction varied from $-7.5 \mathrm{D}$ to $+3.0 \mathrm{D}$ (mean $+0.16(2.6)$ ) in the right eye and from $-8.0 \mathrm{D}$ to $+3.5 \mathrm{D}$ (mean $+0.08(2.6)$ ) in the left eye.

Contrast sensitivity in the VGB monotherapy group varied from 1.5 to 1.95 (mean $1.76(0.14)$ ) in the right eye and from 1.2 to 1.95 (mean $1.74(0.19)$ ) in the left eye. In the CBZ group, the contrast sensitivity varied from 1.65 to 1.95 (mean $1.78(0.11)$ ) in the right eye and from 1.65 to 1.95 (mean $1.78(0.13)$ ) in the left eye. In the control group, the contrast sensitivity varied from 1.65 to 1.95 (mean $1.77(0.14)$ ) in the right eye and from 1.65 to 1.95 (mean $1.8(0.12)$ ) in the left eye. The results of the Pelli-Robson contrast sensitivity tests did not differ significantly between the three groups (ANOVA, Table 1). However, there were four patients in the VGB group who had more than 2 SD reduction in contrast sensitivity compared with the controls $(<1.56$ in the right eye and $<1.49$ in the left eye): (1) two patients with Pelli-Robson 1.50 in both eyes and visual fields in temporal extent $65^{\circ}$ in both eyes, (2) one patient with Pelli-Robson 1.65 in the right eye and 1.20 in the left eye and visual fields in temporal extent $65^{\circ}$ in the right eye and $60^{\circ}$ in the left eye, (3) one patient with Pelli-Robson 1.50 in the right and 1.35 in the left eye and visual fields in temporal extent $45^{\circ}$ in the right and $25^{\circ}$ in the left eye.

Furthermore, there was a significant positive correlation with linear regression between the contrast sensitivity and the temporal extents of the visual fields in VGB monotherapy patients $(\mathrm{R}=0.498, \mathrm{p}=0.005$ in the right eye and $\mathrm{R}=$ $0.476, p=0.006$ in the left eye) (Figs 3 and 4 ). There was no correlation between the contrast sensitivity values and the time of VGB treatment $(R=0.05, p=0.79$ in the right eye and $R=0.11, p=0.56$ in the left eye) or the cumulative dose of VGB $(R=0.20, p=0.30$ in the right eye and $R=0.07, p=0.71$ in the left eye).

The macular photostress test was normal in all patients. In the VGB group 4/62 eyes $(6.5 \%)$ recovered in 30 seconds, the others faster. In the CBZ group, the recovery time for all eyes was less than 30 seconds. In the glare test, there were $5 / 62(8 \%)$ eyes in VGB group 
and 6/36 (16.7\%) eyes in CBZ group where visual acuity with high luminance was two lines lower than the acuity without glare. The others had the same or even better visual acuities than without glare. These results are also within the normal limits.

We examined dark adaptation thresholds in the right eye and only the final threshold in the left eye in seven VGB patients with abnormal visual fields. Only one patient (with the visual field in temporal extent $45^{\circ}$ in both eyes), had slightly abnormal thresholds in the right eye: $5.2 \log$ units for cones and $2.9 \log$ units for rods, and $2.8 \mathrm{log}$ units for the rods in the left eye. The time for both thresholds was normal. The others had normal dark adaptation curves.

\section{Discussion}

The results of this study indicate that CBZ has no effect on contrast sensitivity. VGB seems to impair contrast sensitivity in patients with concentric constriction in visual fields. We found no alterations in glare sensitivity.

The macular photostress test is used in differential diagnosis of macular diseases and optic neuropathies in patients with decreased visual acuity. Patients with macular disturbances have a prolonged recovery time from glare instead of the normal recovery time found in patients with optic neuropathies. ${ }^{24}$ Bayer et $a l^{\beta}$ showed that glare sensitivity was affected in CBZ therapy patients indicating drug induced retinal dysfunction. In the present study, epilepsy patients undergoing CBZ or VGB monotherapy did not show abnormal sensitivity for glare.

We could not confirm the previous results of Tomson et $a l^{2}$ and Sartucci et $a l^{17}$ that CBZ therapy impairs contrast sensitivity of patients with epilepsy. In Sartucci's study, add-on therapy with VGB even seemed to improve impaired contrast sensitivity observed in GBZ therapy patients. Our results show that CBZ therapy has no negative influence on these visual function tests, but that VGB therapy possibly impairs contrast sensitivity.

Pow et $a l^{25}$ observed a transport of immunolabelled $\gamma$-vinyl-GABA (VGB) to GABAergic amacrine cells in the retinas of rabbits, cats, chickens, fish, and monkeys. The increase in the amount of the inhibitory neurotransmitters in the postreceptoral retina allows us to suspect a greater decrease in contrast sensitivity in VGB monotherapy patients. In our study, the VGB therapy patients who had concentric constricted visual fields had lower contrast sensitivity values than those with normal visual fields.

The normal results of macular photostress and glare tests as well as normal visual acuities support the theory that the central retinas and optic nerves of VGB therapy patients are not affected even though the visual fields are concentrically constricted. We do not know the exact mechanism of visual field constriction and low contrast sensitivity in VGB therapy patients. The mechanism could be the same in both, or the concentric constriction in the visual fields may lead to reduction in contrast sensitivity. However, larger series are needed to determine the prevalence of impaired contrast sensitivity in patients undergoing VGB therapy. Different ocular function tests are also needed to identify possible ophthalmic adverse effects in patients receiving antiepileptic drugs.

1 Mattson RH. Carbamazepine. In: Engel J Jr, Pedley TA, ed. Epilepsy. A comprehensive textbook. Vol 2. Philadelphia: Lippincott-Raven, 1998:1491-502.

2 Tomson T, Nilsson BY, Levi R. Impaired visual contrast sensitivity in epileptic patients treated with carbamazepine. Arch Neurol 1988;45:897-900.

3 Bayer A, Thiel HJ, Zrenner E, et al. Farbsinnstörungen und erhöhte Blendungsempfindlichkeit unter Phenytoin- und Carbamazepintherapie. Nervenarzt 1995;66:89-96.

4 Bayer A, Theil HJ, Zrenner E, et al. Empfindliche sinnesphysiologisch Testmethoden für oculäre Nebenwirkungen von Medikamenten am Beispiel verschiedener Antiepileptika. Ophtalmologe 1995;92:182-90.

5 Perez M-TR, Davanger S. Distribution of GABA immunoreactivity in kainic acid-treated rabbit retina. Exp Brain Res 1994;100:227-38.

6 Arnarsson A, Eysteinsson T. The role of GABA in modulating the Xenopus electroretinogram. Vis Neurosci 1997;14: $1143-52$.

7 Davson H. Transmitters in the retina .In: Physiology of the eye. 5th ed. London: MacMillan Press, 1990:343-52.

8 Ben-Menachem E. Vigabatrin. Epilepsia 1995;36:S95-104.

9 Kälviäinen R, Keränen T, Riekkinen PJ Sr. Place of newer antiepileptic drugs in the treatment of epilepsy. Drugs 1993;46:1009-24.

10 Eke T, Talbot JF, Lawden MC. Severe persistent visual field constriction assosiated with vigabatrin. BMf 1997;314: $180-1$.

11 Wong ICK, Mawer GE, Sander JWAS. Severe persistent visual constriction associated with vigabatrin. Reaction may be dose dependent. BMF 1997;314:1693-4.

12 Blackwell N, Hayllar J, Kelly G. Severe persistent visual constriction associated with vigabatrin. Patients taking vigabatrin should have regular visual field testing. BMF 1997;314:1694

13 Kramer G, Scollo-Lavizzari G, Jallon P, et al. Vigabatrinassociated bilateral concentric visual field defects in four patients. Epilepsia 1997;38:179.

14 Mackenzie R, Klistorner A. Severe persistent visual constriction associated with vigabatrin. A symptomatic as well as symptomatic defects occur with vigabatrin. BMF 1998;316:232.

15 Krauss GL, Johnson MA, Miller NR. Vigabatrin-associated retinal cone system dysfunction. Electroretinogram and ophtalmologic findings. Neurology 1998;50:614-18.

16 Kälviäinen R, Äikiä M, Saukkonen A, et al. Vigabatrin vs carbamazepin monotherapy in patients with newly diagnosed epilepsy. Arch Neurol 1995;52:989-96.

17 Sartucci F, Massetani R, Galli R, et al. Visual contrast sensitivity in carbamazepine-resistant epileptic patients receiving vigabatrin as add-on therapy. F Epilepsy 1997;10:7-11.

18 Steinhoff B, Freudenthaler N, Paulus W. The influence of established and new antiepileptic drugs on visual perception II. A controlled study in patients with epilepsy under long-term antiepileptic medication. Epilepsy Res 1997;29: 49-58.

19 Tiel-Wilck K, Jokiel B, Zinser P, et al . Afferent visual function after single dose application of $\gamma$-vinyl GABA. Neuroophtalmology 1995;15:305-10.

20 Steinhoff B, Freudenthaler N, Paulus W. The influence of established and new antiepileptic drugs on visual perception.1. A placebo-controlled, double blind, single-dose study in healthy volunteers. Epilepsy Res 1997;29:35-47.

21 Kälviäinen R, Nousiainen I, Mäntyjärvi, et al. Vigabatrin, a GABAergic antiepileptic drug, causes concentric visual field defects. Neurology 1999;53:922-6.

22 Pelli D, Robson J, Wilkins A. The design of a new letter chart for measuring contrast sensitivity. Clin $\mathrm{Vis} \mathrm{Sci}$ 1988;2:187-99.

23 Rubin G. Reliability and sensitivity of clinical contrast sensitivity tests. Clin Vis Sci 1988;2:169-77.

24 Glaser J, Savino P, Sumers K, et al. The photostress recovery test in the clinical assessment of visual function. Am $\mathcal{F} O p h^{-}$ thalmol 1977;83:255-60.

25 Pow DV, Baldridge W, Crook DK. Activity-dependent transport of GABA analogues into specific cell types demonstrated at high resolution using a novel immunocytochemical strategy. Neuroscience 1996;73:1129-43. 